\title{
Advances in management of subretinal choroidal neovascularization membrane clinically
}

\begin{abstract}
Sub retinal choroidal retinal membrane is a disease of the retina that affects elderly patient with degenerative pathologies. This case outlines a brief sketch of pathogenesis and treatment protocol of this disease in current era. It is known for self-limited and regressive behavior that can be observed thoroughly and typically has a favorable visual prognosis.
\end{abstract}

Keywords: metamorphopsia, RPE, choroidal rupture, central serous chorioretinopathy, spergnitous choroiditis

\author{
Volume 7 Issue 6 - 2017
}

\begin{abstract}
Rubina Shah
King Edward Medical University Lahore/National Eye Center, Pakistan
\end{abstract}

\author{
Correspondence: Rubina Shah, King Edward Medical \\ University Lahore/National Eye Center, Lahore, I IA sandha \\ road National eye center Lahore, Pakistan, Tel 009234240I4487 \\ Email binashah002@gmail.com
}

Received: October 20, 2017 | Published: November 21, 2017

\begin{abstract}
Abbreviations: FA, fluorescein angiography; POHS, presumed ocular histoplasmosis syndrome; NSAIDs, nonsteroidal anti-inflammatory drugs; APMPPE, acute posterior multifocal placoid pigment epitheliopathy; MEWDS, multiple evanescent white dot syndrome, PIC, punctate inner choroidopathy; DSF, diffuse sub retinal fibrosis
\end{abstract}

\section{Introduction}

A 51year old Asian male presented for examination with the complaint of a dark spot distorting his central vision associated with shady and fuzzy lines in right eye even with correction. He denied ocular hyperemia, lacrimation, discharge, pain and headache. His ocular history included presbyopia and glaucoma suspect. He had cataract extracted OU with OD vitrectomized 15years prior. $\mathrm{He}$ denied positive family ocular history. The patient's medical history was positive for diabetes and hypertension. He was using medication for both systemic disease but could not recall his prescription. Social history was negative for tobacco, alcohol, or recreational drug use. He was using Alphagan eye drop but no known drug allergies. He was oriented to time, place, and person and his mood was appropriate.

Uncorrected distance visual acuity was 20/160 OD and 20/40 OS Habitual spectacle correction measured was $-2.75 /-1.25 * 140$ OD and-1.00/-1.25*100 OS, with an ad of +2.50 OU. Manifest spectacle correction revealed change in refraction. Near visual acuity with correction were not recognizable OD and $\mathrm{N}_{5}$ OS. There was evidence of relative afferent pupillary defect with pupils were equal round sluggishly reactive contralateral eye. Confrontation fields were full to finger counting in OS but restriction of visual field in OD. Extraocular muscles were unrestricted in all gazes without pain or diplopia. Cover test was orthophoric at distance and near. Amsler Grid test effectively depicted central scotoma with metamorphopsia. Goldman applanation tonometry was $14 \mathrm{mmHg}$ OD, $13 \mathrm{mmHg}$ OS at $12: 10 \mathrm{pm}$. Slit lamp biomicroscopy revealed normal adnexa. Bilateral cornea was normal transparency and demonstrated arcus senilis. Both anterior chambers appeared quiet without evidence of cell or flare estimation of the chamber angles was $4 / 4$ via the Von Herrick method. Both irides were flat and brown. Pupils were dilated using one drop 1\% Mydriacyl. Evaluation of the posterior segment revealed bilateral pseudophakic. Fundus assessment revealed pale disc with parapapillary atrophy of optic nerve head. The cups were deep and there was no evidence of edema in the neuroretinal rim. Vitreous shows bleed due to extension of blood from retina. It affected central vision as right macula visualized sub macular hemorrhage with hard yellow exudates in retina, chorioretinal atrophy with cystoids macular odema.

Fluorescein angiography (FA) is vital to assess the disease. It helps to determine the location, extent, definition of the disease and its relation to center of fovea for the treatment implications. SRNVM can be divided according to angiographic appearance into those membranes that are well-defined and those that are poorly defined (Figure 1).

Major differential diagnoses to be considered are:

i. Angioid Streaks

ii. Birdshot Retinopathy

iii. Central Serous Chorioretinopathy

iv. Degenerative myopia

v. Presumed Ocular Histoplasmosis Syndrome

vi. Psuedophakic (Irvine-Gass) Macular Edema unlikely 15year status post-operative

vii. White Dot Syndromes

Angioid streak are the result of crack like dehiscence, calcified brittle collagenous elastic portion of Bruch's membrane. ${ }^{1,2}$ It is known as ("Peau d"e orange) orange skin consisting of molting skin pigmentation. Linear, greyish lesion with irregular edge lies beneath blood vessels and intercommunicate with optic disc and peripheral area of retina. They are commonly associated with optic disc drusen. Visual impairment occurs over $70 \%$. The prognosis can be guarded cautiously because any of the following can result 1) Choroidal neovascularization 2) Choroidal rupture 3) foveal involvement. Photocoagulation can be successful treatment.

Central Serious Chorioretinopathy is characterized by localized detachment of sensory retina secondary to RPE and choroid defects due to breech in retina caused by accumulation of fluid. The major cause that aggravates CSR includes stress, untreated hypertension, alcohol use, organ transplant and administration of steroids. ${ }^{3-5}$ Fundoscopy shows RPE lesions with prominent elevation of the 
neurosensory retina by clear fluid typical of cases of recent onset to shallow detachments overlying large patches of irregularly depigmented RPE.The treatment is based largely on observation. The high spontaneous remission rate favors conservative management, lifestyle counseling, and discontinuation of glucocorticoid medication as first-line therapeutic options. The strategy can be expected to be followed by a resolution of the detachment in nearly $90 \%$ of cases within 1.5 months.

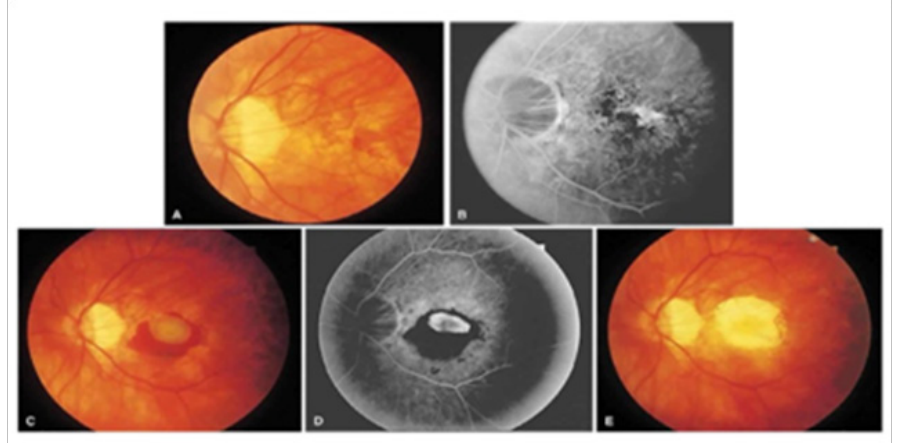

Figure I Illustrates FFA of posterior segment with SRCNVM.

Myopia is highly associated with degenerative changes in the posterior segment of the eye that can be degenerative or pathological myopia. ${ }^{4}$ It can result in abnormal visual function, such as a decrease in best corrected visual acuity or changes in visual fields. Complications such as retinal detachment and glaucoma are relatively common. ${ }^{5}$. Patients with degenerative myopia should be advised to have annual or more frequent examination of eye depending upon the severity of ocular changes. Clinicians should educate them about the causes and symptoms of retinal break or detachment and glaucoma or CNV. Patients should also be advised to seek prompt care if they experience the onset of symptoms. Patients with degenerative myopia should be instructed to avoid the causes of blunt trauma and to wear eye protection when they are at risk for blunt ocular trauma (e.g., playing tennis, racquetball).

\section{Presumed ocular histoplasmosis syndrome}

(POHS) is choroidopathy typically characterized by atrophic chorioretinal scar. The ocular manifestation presumably shows a fungal infection which includes peripapillary atrophy, peripheral punchedout lesions, and a macular sub retinal neovascular membrane. ${ }^{6,7}$ Laser photocoagulation can be beneficial in reducing visual loss from macular scarring due to sub retinal neovascular membranes. Vision improved from $20 / 70$ to $20 / 20$ in the treated eye.

\section{Birdshot retinochoroidopathy}

It is a rare, chronic, bilateral, posterior uveitis with a clinical presentation and a strong genetic association. The pathogenesis is unknown, but HLA-A29 positivity appears to confer predisposition and retinal autoimmunity seems to plays a major role. ${ }^{8}$ Patient appears with blurry vision experiencing floaters, dyschromatopsia, light sensitivity, metamorphism and decreased peripheral vision. HLA-29 antigen which is present $80-90 \%$ controls the risk factors of disease occurrence. ${ }^{9}$ The treatment option is considered unless visual acuity reduces bilaterally $20 / 40$ due to cystoid macular oedema. One notable exception to this rule applies to sub retinal neovascularization threatening central vision. The treatment requires prompt laser treatment with periocular and systemic steroidal therapy. Periocular steroid are used for diseases with asymmetric severity and for exacerbations in patients on systemic therapy. Cyclosporine is effective because it compromises CD4 lymphocyte function, which is thought to mediate birdshot.

\section{Pseudophakic macular edema (Irvine-Gass syndrome)}

The most common causes of visual loss after cataract surgery are PMO not after 15years. ${ }^{10,11}$ The latest technique phacoemulsification has drastically reduced pseudophakic CME incidence with small incision cataract surgery but pseudophakic CME remains a common complicate morbidity because it is most common surgery. The management of pseudophakic CME is based on its pathogenesis. It is associated with retinal vein occlusion (RVO), epiretinal membrane (ERM), and prostaglandin analogs. ${ }^{12}$ Several clinical trials and cellular studies presented preoperative and postoperative topical glaucoma medications, specifically latanoprost and timolol may increase the incidence of pseudophakic CME. ${ }^{13}$ The importance of corticosteroids reduces inflammation by inhibiting phospholipase A2, and nonsteroidal anti-inflammatory drugs (NSAIDs) inhibit COX. The 2 critical COX isoforms are COX-1 and COX-2, and the latter is the major isoform expressed in the retina. ${ }^{14}$ The treatment option is topical corticosteroids, Periocular ocular corticosteroids, intraocular triamcinolone, avastin therapy and surgically pars plana vitrectomy. ${ }^{15,16}$

\section{White dot syndrome}

The white dot syndromes are a group of idiopathic multifocal inflammatory conditions involving the retina and the choroid. They are characterized by the appearance of white dots in the fundus. The diseases that constitute the white dot syndromes include acute posterior multifocal placoid pigment epitheliopathy (APMPPE), serpiginous choroiditis, multiple evanescent white dot syndrome (MEWDS), multifocal choroiditis and panuveitis (MCP), punctate inner choroidopathy (PIC), and diffuse sub retinal fibrosis (DSF).

\section{Management plan}

The management option was considered when it caused vision deterioration due to bleed involving macula and central vision. Initially the disease was variable and observation helped for small nonexudative bleeding. The treatment outlines the extent and location of SRNVM. When there is fluid accumulation with serous separation the treatment should not create a white reaction in the retina. ${ }^{17}$ Adequate and successful treatment with photocoagulation limits the size of a SRNVM and aborts its growth potential. It includes laser photocoagulation, photodynamic therapy, macugen, lucentis and avastin injections. Recently, pharmacotherapy with anti-angiogenic drugs has markedly changed our approach and expectations in the management of sub retinal neovascularization. The choice for vitreoretinal medical community has been intravitreal anti-VEGF injections that target the underlying cause of CNVM in the "wet" form of macular degeneration. The abnormal blood vessels found in CNVM reacts to the medication by "drying up" after multiple injection applications. Its result has been very encouraging for macular degeneration patients.

However, clinically evidence of the peripheral peripapillary SRNVM may cause irreversible threatening of vision due to of bleeds in sub fovea area and may affect bilaterally with ratio of $75 \% .^{18,19}$ Peripapillary SRNVM often results in very large complexes before they become symptomatic. Although, macular laser can be beneficial, which shows a $50 \%$ recurrence rate $?^{20}$ Certainly, literature hypothesized surgical pars plana vitrectomy could completely remove the SRNVM complex, possibly decreasing the recurrence 
rate and sparing the papillomacular bundle from further damage. Recent studies has shown an effective advancement in treatment with intravitreal injections of recombined tissue plasminogen activator (rtPA), expansive gas and bevacizumab in eyes with acute sub retinal hemorrhage (SRH) has improved patient visual prognosis of $35 \%$ after 3 month. ${ }^{21}$.

\section{Follow Up\# I}

Patient was advised for intravitreal Avastin Injection with 4-6weeks follow up examination with repetition of diagnostic test OCT. Patient initially recovered $20 / 80$ or better vision confirming after 3-6weeks the ability of Avastin injection (ranibizumab) showed good outcome that reduced neovascularization of $45 \%$. Additionally, there was only a $17 \%$ recurrence rate within the first 1 year, indicating a lower recurrence rate than noted with macular laser.

\section{Follow\#2}

Surgical therapy was considered by ophthalmologist with antiangiogenic therapy due cumbersome of follow up therapy for avastin injection. Pars plana vitrectomy with fluid gas exchange was performed to remove SRNVM. The exudates resolved by visual acuity 20/40. Follow up examination after six month was scheduled to observe recurrence.

\section{Discussion}

SRNVM is a sight threatening disease that causes degenerative changes in macula involving development of leaking vessels with neovascularization. SRNVM's have been observed idiopathically in both young and old patients. They are also seen in patients with senile (exudative) macular degeneration, presumed ocular histoplasmosis syndrome, severe myopia, chorioretinal atrophy resulting from healed disseminated chorioretinitis of unknown etiology, toxoplasmosis, toxocariasis, sarcoidosis, drusen of the optic nerve head hereditary drusen of Bruch's membrane, angioid streaks, pseudotumor cerbri, choroidal folds, fundus flavimaculatus, choroidal melanomas, harada's disease, acute multifocal posterior placoid pigment epitheliopathy, Behcet's disease, geographic helicoid (serpiginous) choroiditis and choroidal rupture. SRNVM's may also occur after excessively intense photocoagulation accumulation..$^{22,23}$

The histopathology shows stages of accumulation where the retinal pigmented epithelium and sensory retina may be elevated either by plasma or hemorrhage. Clinically chronic manifestations may be represented by subretinal hemorrhage, reabsorbing subretinal hemorrhage which is often yellow in color, hard yellow exudates in the retina, cholesterol crystals, subretinal fibrosis, chorioretinal atrophy, clumping of melanin granules, areas of hypo and hyperpigmentation, chronic serous separation of the central retina and overlying cystoid macular edema. Hemorrhage from a SRNVM breaks through into the vitreous obscuring fundus details. In the chronic stages one observes subretinal fibrosis, which is largely the residual mesenchymal tissue accompanying the growth of the neovascular component. Some subretinal fibrosis is the result of metaplasia of the retinal pigmented epithelium. This chronic serous of sometimes hemorrhagic separation of the sensory retina predisposes to continuous atrophy of the photoreceptors and a deleterious effect on the retinal pigmented epithelium. As a consequence of chronic serous separation of the retina there may also be dilation and tortuosity of the overlying retinal vessels. ${ }^{24-27}$

The history of management of extra foveal SRNVM in 2005 which was initially published in cannes retina annual meeting of American society of Vitroretina founded unsuccessful outcome from sub retinal surgery. ${ }^{28}$ Hence hypothesized removal of extrafoveal SRNVM would possibly spare the foveal retinal pigment epithelium (RPE). The success rates were only a $17 \%$ recurrence rate within the first 3years, indicating a lower recurrence rate than noted with macular laser. At final follow-up (i.e. 3 to 6years following surgery), $83 \%$ of patients improved one or more lines. ${ }^{29,30}$

\section{Conclusion}

Sub retinal choroidal retinal membrane is a degenerative, selfremitting and regressive disease of retina that produces abnormal leaking vessels in vital layers of retina which compromise the integrity of retina affecting functionality of blood retinal barrier. It causes central deterioration of vision. The progression of the SRNVM ophthalmoscopically or angiographically might be an indication for laser treatment. Once subfoveal extension occurs, the visual outcome tends to be unfavorable. No patients had recurrence if papilledema regresses.

\section{Funding details}

None.

\section{Acknowledgements}

None.

\section{Conflicts of interest}

Authors declare that there is no conflict of interest.

\section{References}

1. Shields JA, Federman JL, Tomer TL, et al. Angioid streaks. I. Ophthalmoscopic variations and diagnostic problems. Br J Ophthalmol. 1975;59(5):257-266

2. Lim JI, Bressler NM, Marsh MJ, et al. Laser treatment of choroidal neovascularization in patients with angioid streaks. $\mathrm{Am} \mathrm{J}$ Ophthalmol.1993;16(4):414-423

3. Gemenetzi M, De Salvo G, Lotery AJ.Central serous chorioretinopathy:an update on pathogenesis and treatment Eye (Lond). 2010;24(12):17431756.

4. Goss DA, Eskridge JB. In:Amos JF, editor. Diagnosis and management in vision care. USA, Boston:Butterworths; 1987. pp. 121-171.

5. Karakus SH, Basarir B, Pinarci EY, et al. Long-term results of half-dose photodynamic therapy for chronic central serous chorioretinopathy with contrast sensitivity changes. Eye. 2013;27(5):612-620.

6. Curtin BJ. The myopias:basic science and clinical management Philadelphia:Harper \& Row. 1985;31(5):357-358.

7. Nussenblatt RB, Whitcup SM, Palestine AG. Ocular histoplasmosis. In:Nussenblatt RB et al. editors. Uveitis:fundamentals and clinical practice, 2nd edn. Mosby, USA:St. Louis; 1985.

8. Brezin AP, Monnet D, Cohen JH, et al. HLA-A29 and birdshot chorioretinopathy. Ocul Immunol Inflamm. 2011;19(6):397-400.

9. Zucchiatti I, Miserocchi E, Sacconi R, et al. HLA-A29-Positive Uveitis:Birdshot Chorioretinopathy, What Else? Case Rep Ophthalmol. 2013;4(3):287-293.

10. Johnston RL, Mitchell PC, Berman AM. Omini eye services of Wilmington DE 19808. Journal of American optometric Association. 1988;59(5):401-405 
11. Shelsta HN, Jampol LM. Pharmacologic therapy of pseudophakic cystoid macular edema: 2010 update. Retina. 2010;31(1):4-12.

12. Henderson BA, Kim JY, Ament CS, et al. Clinical pseudophakic cystoid macular edema. Risk factors for development and duration after treatment. J Cataract Refract Surg. 2007;33(9):1550-1558.

13. Miyake K, Ibaraki N, Goto Y, et al. ESCRS Binkhorst lecture 2002: Pseudophakic preservative maculopathy. J Cataract Refract Surg. 2003;29(9):1800-1810.

14. Flach AJ. The incidence, pathogenesis and treatment of cystoid macular edema following cataract surgery. Trans Am Ophthalmol Soc. 1998;96:557-634.

15. Miyake K, Ibaraki N, Goto Y, et al. ESCRS Binkhorst lecture 2002:Pseudophakic preservative maculopathy. J Cataract Refract Surg. 2003;29(9):1800-1810.

16. Arevalo JF, Garcia-Amaris RA, Roca JA, et al. Primary intravitreal bevacizumab for the management of pseudophakic cystoid macular edema:pilot study of the Pan-American Collaborative Retina Study Group. J Cataract Refract Surg. 2007;33(12):2098-2105.

17. Fine SL, Patz A, Orth DH, et al. Subretinal neovascularization developing after prophylactic argon laser photocoagulation of atrophic macular scars. Amer J Ophthalmol. 1796;82(3):352-357.

18. Yonekawa Y, Kim IK. Pseudophakic cystoid macular edema. Curr Opin Ophthalmol. 2012;23(1):26-32.

19. Lopes PF, Green WR. Peripapillary subretinal neovascularization. A Review. Retina. 1992;12(2):147-171.
20. Kies JC, Bird AC. Juxtapapillary choroidal neovascularization in older patients. Am J Ophthalmol. 1988;105(1):11-19.

21. Michelson JB, Michelson PE, Chisar FV. Amer J Ophthalmol. 1980:90.

22. Mayer $\mathrm{CH}$, Scholl HP, Eter N, et al. Combined treatment of acute subretinal haemorrhages with intravitreal recombined tissue plasminogen activator, expansile gas and bevacizumab:a retrospective pilot study. Acta Ophthalmologica. 2008;86(5):490-494.

23. Morse PH, Leveille AS, Antel JP, et al. Bilateral juxtapapillary subretinal neovascularization associated with pseudotumor cerebri. Amer J Ophthalmol. 1981;91(3):312.

24. Smith RE, Kelley JS, Harbin TS. Late macular complications of choroidal ruptures. Amer J Ophthalmol. 1974;77(5):650-658.

25. Morse PH, Leveille AS, Antel JP, et al. Bilateral juxtapapillary subretinal neovascularization associated with pseudotumor cerebri. Amer $J$ Ophthalmol. 1981;91(3):312.

26. Fine SL. Perspectives in Ophthalmology. 1977.

27. Schloegel TF. Perspectives in Ophthalmology. 1977.

28. Shcatz H, Patz A. Exudative senile maculopathy. II. Complications of argon laser treatment. Arch Ophthalmol. 1973;90(3):197.

29. Flaxel CJ, Bird AC, Hamilton AM, et al. Partial laser ablation of massive peripapillary subretinal neovascularization. Ophthalmology. 1996;103(8):1250-1259.

30. Kies JC, Bird AC. Juxtapapillary choroidal neovascularization in older patients. Am J Ophthalmol. 1988;105(1):11-19. 